\title{
COMPARING WORKLOAD BASED ORDER RELEASE MECHANISMS
}

\author{
N.O. Fernandes \\ Instituto Politécnico de Castelo Branco \\ nogf@est.ipcb.pt \\ S. Carmo-Silva \\ Universidade do Minho \\ scarmo@dps.uminho.pt
}

\begin{abstract}
A variety of orders release mechanisms have been developed for workload control. However, in many situations it is difficult to fully understand the behaviour of these mechanisms due to its complexity. In this paper order release mechanisms are compared by studying the influence of single release strategies. Simulation results show that real world order release mechanisms are likely to benefit from incorporating the workload balancing and the atemporal workload accounting over time strategies.
\end{abstract}

\section{INTRODUCTION}

Workload Control (WLC) is a Production Planning and Control (PPC) concept, particularly appropriate for jobbing and flow shops in the make-to-order (MTO) sector of industry (Haskose et al., 2004). It has received much attention both from researchers and practitioners alike, particularly due to its simplicity and similarity to what is done in practice. WLC is an approach applied to control workload in the shop floor. The main principle is to keep the length of queues on the shop floor at appropriate levels to meet the promised deliver dates, taking into account the system capacity and capabilities.

Orders release is a main control element within WLC. It determines the moment and the orders (jobs) to release into the shop floor. Orders arrive from customers over time but they are not immediately released, rather they are collected in a pre-shop pool. The collected orders are assessed periodically and are only released if they fit workload norms for the required capacity groups (e.g. machines). The decision to release an order is usually based on its urgency and influence on the current shop floor situation (Henrich et al., 2004). Once released, a job remains in the shop floor until all of its operations have been completed. Priority dispatching rules determines which orders or jobs in queue, should be selected next for processing in a resource or machine that becomes available. This clearly influences the progress of individual orders through the shop floor. Due to easy handling and general industrial acceptance, a variety of such rules have been devised for application in the shop floor. Ramasesh (1990) makes a review on this topic.

Several order release mechanisms have been developed for workload control. Graves et al. (1995), Bergamaschi et al. (1997) and Fowler et al. (2002) review literature on this matter. Two of the most known mechanisms proposed in the literature are the Load Oriented Order Release (LOOR) (Bechte, 1988) and the Lancaster University Management School (LUMS) approach (Hendry and Kingsman, 1991, Stevenson and 
Hendry, 2006). However, until now no mechanism has shown to be the best for order release and production control over a wide range of conditions. This is due partly to the following:

(1) Order release mechanisms performance highly depends on production control conditions, such as dispatching rules and shop load;

(2) The shop floor operation conditions, i.e. type of manufacturing system, processing times variability, due date tightness when set externally (e.g. by the customer), and machine unavailability, can have a major impact on the mechanisms overall performance; and

(3) Different mechanisms have been evaluated by addressing them as a whole, rather than by setting reference to their inherent structure. This remark have leaded Cigolini and Portioli (2002) to suggest that, comparative analysis should be performed by considering two or more order release mechanisms not as a whole, but by switching single features, each of them related with different characteristics of the release procedure.

To this respect Bergamaschi et al. (1997) classifies order release mechanisms based on eight dimensions that describe the fundamental principles, characteristics and logic of the mechanisms. In this paper, we adopted the above methodology comparing order release mechanisms that only differ by the strategies used in the workload control and the workload accounting over time dimensions. In particular we studied the influence two typical shop configurations on the performance of these strategies. The results of this study should contribute to the choice of the appropriate order release mechanism in practical situations.

The remainder of the paper is outlined as follows: section 2 addresses the overall research methodology and introduces the general structure of the order release strategies tested; section 3 presents, analyses and discusses the results of the simulation study; and finally in section 4 , concluding remarks are made and directions for future research work are presented.

\section{RESEARCH METHODOLOGY}

In order to evaluate the influence of the shop configuration on order release strategies behaviour, a simulation study was carried out using Arena software. During simulation runs, data were collected with reference to the system steady state. The simulation runs last for 27600 time units. For each simulation run 90 independent replications were performed. To avoid initialization bias a warm-up period of 9600 time units was used. Common random numbers were used as a variance reduction technique across all experiments.

This section details the simulation model and the release strategies tested.

\subsection{Simulation Model}

A job shop without an explicit bottleneck has been the starting point of this investigation. The job shop consists of six work centres each containing a single multi-purpose machine. In the simulation model, the job's routing is randomly chosen from a set of twenty routings each of which with an equal probability of occurrence, see Table 1.

Because most real life job shops exhibit a prevalent flow pattern, Enns (1995) argues that these shops have most in common with the theoretical general flow shop. So, a general flow shop was also considered in the simulation study. Routings for this shop are established in a way similar, only with work centres being visited in order of increasing 
work centre number. This ensures that the flow between any combinations of two work centres will always have the same direction, as required in general flow shops.

Table 1. Work centre job routing matrix

\begin{tabular}{|c|c|c|c|c|c|c|}
\hline \multirow{2}{*}{$\begin{array}{l}\text { Routing } \\
\text { pattern }\end{array}$} & \multicolumn{7}{|c|}{ Operation number } \\
\hline & 1 & 2 & 3 & 4 & 5 & 6 \\
\hline 1 & 2 & 4 & 6 & 1 & 5 & 3 \\
\hline 2 & 1 & 3 & 5 & & & \\
\hline 3 & 2 & 3 & 5 & 4 & & \\
\hline 4 & 5 & & & & & \\
\hline 5 & 4 & 2 & 5 & 6 & 1 & \\
\hline 6 & 2 & 5 & 4 & 6 & 1 & 3 \\
\hline 7 & 1 & 3 & 2 & 6 & & \\
\hline 8 & 2 & 6 & & & & \\
\hline 9 & 2 & 5 & 4 & & & \\
\hline 10 & 3 & 1 & 5 & 4 & 6 & 2 \\
\hline 11 & 6 & 2 & 3 & & & \\
\hline 12 & 2 & 6 & 1 & 3 & 2 & \\
\hline 13 & 2 & 3 & 6 & & & \\
\hline 14 & 4 & 1 & 2 & 5 & 3 & \\
\hline 15 & 1 & & & & & \\
\hline 16 & 4 & 3 & 6 & 5 & 1 & \\
\hline 17 & 4 & & & & & \\
\hline 18 & 3 & 4 & 6 & 5 & & \\
\hline 19 & 4 & 1 & 6 & & & \\
\hline 20 & 4 & 1 & & & & \\
\hline
\end{tabular}

As a result of the number of operations in each of the twenty routings indicated in Table 1, the mean number of operations per routing is 3.6. Processing times for all machines are identical, following a 2-Erlang distribution with a mean of 1 time unit. According to Oosterman et al. (2000) the 2-Erlang distribution approaches well the observations made in real life job shops. An average planned system utilization of $90 \%$ is ensured by setting the appropriate time between jobs arrivals. An exponential distribution is used for the jobs inter-arrivals times, as this typically explains the stochastic nature of job arrivals.

Due dates are assigned to jobs on their arrival and are modelled as a random variable. They are established to ensure that, under immediate release, the number of tardy jobs falls between $5 \%$ and $10 \%$. This is determined by the jobs arrival time plus a uniformly distributed allowance between 50.8 and 60.8 time units. After the assignment of the due date, jobs are placed in the pre-shop pool, waiting for release. Job release decisions are made periodically, every 8 time units, in the beginning of each release period. Each job is considered for release according to the earliest planned release time. Planned release times are determined by backward scheduling from the due date, using the work centres lead times, as follows:

$$
\mathrm{r}_{\mathrm{j}}=d_{j}-\sum_{s \in S_{j}} L T_{s}
$$

Where $\mathrm{LT}_{\mathrm{s}}$ is the work centre lead time, $\mathrm{r}_{\mathrm{j}}$ is the planned release time of job j, $\mathrm{d}_{\mathrm{j}}$ is the planned job $\mathrm{j}$ due date and $S_{\mathrm{j}}$ is the set of work centres in the job's routing. Planned work centre lead times were established through some pilot simulation runs, also using immediate release. 
The order release mechanism ensures that a job candidate for release is released only if it fits the established workload norms for the required capacity groups. Every job in the pool with a planned release time within a time limit is a candidate. However, it has been shown (Land, 2006) that imposing such a time limit tend to deteriorate the performance of order release mechanisms. Thus, in this study no time limit is placed on the release of jobs.

Released jobs follow a first-in-first-out (FIFO) dispatching rule on the shop floor in all work centres. Setup times have been considered sequence independent and assumed as part of the operation processing time.

\subsection{Order release strategies}

Two strategies for workload accounting over time and three for workload control were simulated. The strategies simulated were chosen because they are frequently used and considered to be important in several order release mechanisms (Oosterman et al., 2000, Cigolini and Portioli, 2002).

Workload accounting over time, defines the method of accounting the load of a released job, establishing when and how much of this load should be allocated to each work centre or capacity group. Workload accounting is simulated at two levels or strategies: (1) atemporal and (2) probabilistic.

The probabilistic strategy, accounts for the actual direct load of a work centre (the quantity of work resulting from jobs queued and being processed at the work centre) at the time of release and estimates the input to this load during the release period, resulting from jobs at upstream work centres, using an estimation method called load conversion (Bechte, 1988). This method is detailed by Breithaupt et al. (2002).

Under an atemporal strategy a released job is assumed to instantaneously add up load to each work centre or capacity group on the basis of the job processing time. This means that a job is included in the accounted load of a work centre upon release, and excluded as soon as the operation at that work centre is concluded, thus considering the upstream load (the quantity of work resulting from jobs queued and being processed at upstream work centres) in the same way as the direct load. The accounted load under this strategy is based on adjusted aggregated load method (Oosterman et al., 2000).

Workload control influences job release decisions in order to maintain the load on the shop floor under control. Workload control is simulated at three levels or strategies: (1) upper bound, (2) lower bound and (3) workload balancing.

Under an upper bound workload control, the release of a job to the shop floor is allowed only if workload in all work centres of a job routing does not exceed an upper limit. This means that a job will not be released if, as a result, at least in one work centre of the job routing the workload becomes larger than the upper workload limit established.

The lower bound workload control seeks to avoid 'starving' of work centres by ensuring that workload in all the work centres is above the lower limit. This means that a job will be released if at least in one work centre in the job routing the workload is lower than the lower workload limit.

Workload balancing releases a job only if it contributes to a better load balancing among work centres even if the upper workload limit is exceeded. However, the load at each work centre in the job's routing is not allowed to exceed the upper limit in more than $20 \%$. This $20 \%$ value was established after some pilot simulation runs, ensuring good results. The workload balancing measure employed was the follow index (BI):

$$
B I=\frac{\sum_{i} F_{i j}}{\max _{i} F_{i j} m} \quad(i=1, \ldots, m)
$$


Where $\mathrm{F}_{\mathrm{ij}}$ represents the accounted workload on work centre $i$ resulting from releasing job $j$ into the shop floor, and $m$ represents the number of work centres. As can easily be guessed the best balancing situation is obtained when BI equals one.

\section{SIMULATION RESULTS AND DISCUSSION}

This section presents and discusses the results of the simulation study described in the preceding section. We recorded two main performance measures, namely shop flow time and time in system. The former is defined as the time that elapses between job release and job completion. The latter is defined as the time a job spends waiting in the pre-shop pool plus the shop flow time.

Figures 1 and 2 shows the time in system behaviour for each one of the strategies tested. Figure 1 shows the results for the job shop configuration. Figure 2 shows the results for the general flow shop. In these figures, the average value of the time in system is plotted against the average value of the shop flow time. Superior strategies yield a lower time in system for a given shop flow time, i.e. will have a curve which is shifted down and to the left. A point on the curve is the result of simulating an order release strategy at a specific workload level or norm.

For each of the release strategies, appropriate values for the workload norms, have to be determined. In particularly, we want to compare the strategies based on the time in system at different levels of norm tightness. However, norm levels for each of the strategies cannot be compared directly, because they result in different shop flow times. So we use the shop flow time as an intermediate variable. According to Oosterman et al. (2000) norms of two mechanisms or strategies are equally tight, if they result in the same shop flow time.

As can be seen, curves converge at the higher values of the shop flow time. This is the result of an infinite workload norm level, i.e. the upper or lower limits, according to the workload control strategy, are both very large. As could be expect all strategies give the same results if release is not restricted by workload norms, as it happens in this case for very large values of workload norm levels. However, as norms get particularly tighter, i.e. as shop flow time decreases below a certain point, time in system tends to increase substantially. To some extent, the waiting time in the shop floor tend to be replaced, by the waiting time in the pre-shop pool of orders. However, since the time in system is the sum of the pool time and the shop floor time, we can conclude that waiting times in the pool increase more than waiting times in the shop floor decrease. 


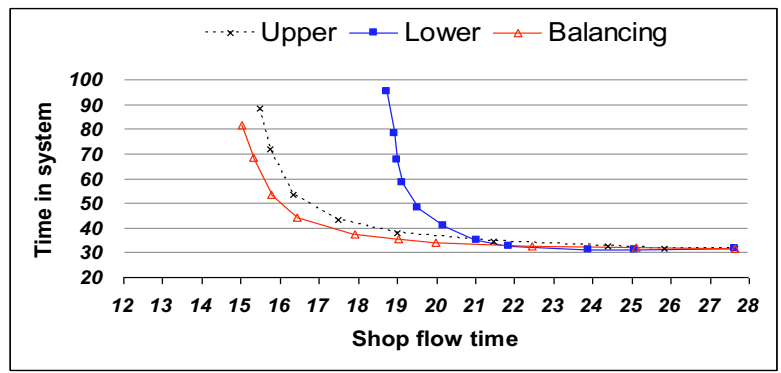

Probabilistic

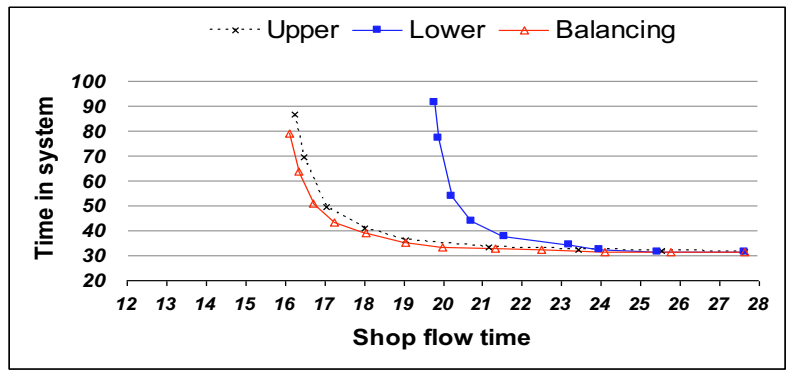

Atemporal

Figure 1. Performance curves for the job shop

Analysis of results of the job shop configuration leads following conclusions.

First, the lower bound workload control strategy does not lend itself to as good control as the upper and balancing strategies do. In fact, it cannot achieve the shop flow time reduction that the others can. This holds for both, atemporal and probabilistic workload accounting strategies.

Second, workload balancing performs slightly better than the upper bound control strategy under both, probabilistic and atemporal strategies, particularly for tighter workload norms, i.e. low values of time in system.

Third, the performance of the workload accounting strategies seems not to be independent of the workload control strategy adopted. Comparing strategies at a shop flow time of 19 time units (i.e. about a 31\% reduction of shop flow time relatively to unrestricted periodic release), we may see that under the lower bound workload control, the probabilistic approach performs visibly better than the atemporal one. However, under the balancing or the upper bound workload control strategies, the probabilistic approach performs similarly to the atemporal one. 


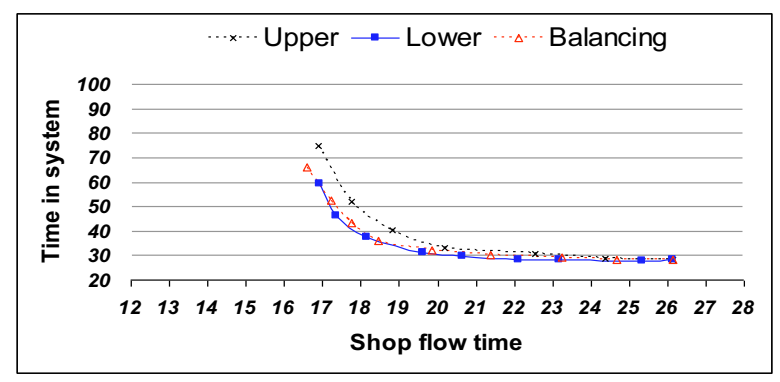

Probabilistic

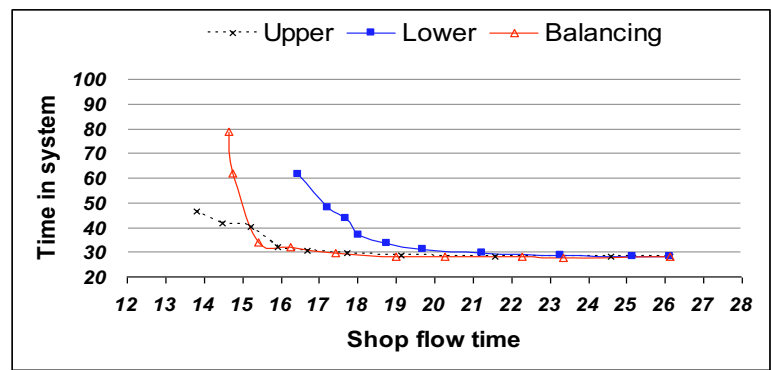

Atemporal

Figure 2. Performance curves for the general flow shop

Analysis of results for the general flow shop case leads to the following conclusions.

First, the performance of the probabilistic strategy deteriorates in the presence of direct work flow, typical of flow shops, both under the upper bound and workload balancing strategies. This can be observed comparing figures 1 and 2.

Second, the performance of the lower bound workload control strategy clearly improves for both, atemporal and probabilistic workload accounting strategies in relation to the job shop case. As work flow becomes more direct, operating differences between workload control strategies become less visible, and similar results may be expected.

Third, consistently with the observation made in the job shop configuration, performance of the workload accounting strategies does not seem to be independent of the workload control strategy adopted.

Fourth, it is not evident that workload balancing improves order release compared with the upper workload control strategy. We may explain this by the fact that as job routing becomes more direct, fewer jobs can be found in the pool that contributes to improve the balance index (BI), reason why the two strategies perform similarly. Note that in our simulation processing times for all machines are identical and balancing opportunities become exclusively dependent on jobs routings.

\section{CONCLUSIONS}

Order release mechanisms are strategically important for the economic success of companies having a great influence on system operations performance.

In this study computer simulation was used for evaluating the behaviour of several order release strategies related with workload control and workload accounting over time, instead of evaluating order release mechanisms as a whole. Results are based on a job shop and a general flow shop.

Two important conclusions could be drawn. Frist, even if results show that no single workload control strategy performs best in any condition, overall results show that 'workload balancing' is the best overall performer while the 'lower bound' is the worst. 
Second, performance of the workload accounting strategies does not seem to be independent of the workload control strategy adopted. Results also suggest that, incorporating 'atemporal' workload accounting over time in combination with 'workload balancing' in order release mechanisms used in practice, it is likely to offer good system operating performance.

Although some insights point out the utility of the study for practical use, it is important to extend it to a wider spectrum of order release strategies and dimensions in order to get an in dept understanding of the full complexity behind order release mechanisms.

\section{REFERENCES}

1. Bechte W. Theory and practice of load-oriented manufacturing control. International Journal of Production Research 1988; 26(3), 375-395.

2. Bergamaschi D, Cigolini R, Perona M, Porioli A. Order review and release strategies in a job shop environment: a review and classification. International Journal of Production Research 1997; 35(2): 339420.

3. Breithaupt JW, Land J, Nyhuis, P. The workload control concept: theory and practical extensions of Load Oriented Order Release. Production Planning and Control 2002; 13(7): 625-638.

4. Cigolini R, Portioli A. An experimental investigation on workload limiting methods within ORR policies in a job shop environment. International Journal of Production Research 2002; 13(7): 602-613.

5. Fowler JW, Hogg GL, Mason, SJ. Workload control in the semiconductor industry. Production Planning and Control 2002; 13(7): 568-578.

6. Graves RJ, Konopka JM, Milne RJ. Literature review of materials flow control mechanisms. Production Planning and Control 1995; 6(5): 395-403.

7. Haskose A, Kingsman BG, Worthington, D. Performance analysis of make-to-order manufacturing systems under different workload control regimes. International Journal of Production Economics 2004; 90(2): $169-186$

8. Hendry L, Kingsman BG. A decision support system for job release in make-to-order companies. International Journal of Operations and Production Management 1991; 11: 6-16.

9. Henrich P, Land M, Gaalman GJC. Exploring applicability of the workload control concept. International Journal of Production Economics 2004; 90(2): 187-198.

10. Land, M. Parameters and sensitivity in workload control. International Journal of Production Economics 2006; 104(2), 625-638.

11. Oosterman B, Land M, Gaalman GJC. The influence of shop characteristics on workload control. International Journal of Production Economics 2000; 68: 107-119.

12. Ramasesh R. Dynamic Job shop scheduling: a survey of simulation research. Omega 1990; 18(1): 43-57.

13 Stevenson M, Hendry L. Aggregated Load-oriented workload control: A review and re-classification of a key approach. International Journal of Production Economics 2006; 104(2): 676-693. 